IJASC 14-1-2

\title{
The Convergence of Life and Technology from the Nurturing Perspective
}

\author{
Robert J. Wierzbicki \\ University of Applied Sciences Mittweida, Germany \\ robert@wierzbicki.org
}

\begin{abstract}
Upbringing requires an active intervention in the process of children growing up. Convergence in technology and media makes it possible to augment nurturing by making blended infrastructures with new digital resources available in learning environments at schools. A game-based provision of educationally-valuable content within a collective, virtual environment combined with an analysis of events in the game after it has been played (blended nurture environment) may help to better communicate human values especially where old-fashioned discussion methods fail or do not fulfill the promise of effective, educational institution-based methods of bringing up. This is where life sciences, psychology, media, pedagogy and technology (need to) converge.
\end{abstract}

Keywords: convergence, life, technology, evolution, digital tool-maker, nurture, nature, blended nurture, gamification, games, behavior

\section{Introduction}

When referring to convergence, the focus is primarily on technologically oriented aspects. These may be physical signal transmission over networks, technical protocols or communication channels sharing the same transmission bandwidth for example. Discussing convergence these days it seems reasonable to shift the point of view towards life and technology convergence and talk about the merging of life sciences and digital experiences rather than consider convergence from a purely technological perspective.

\section{The digital Tool-maker and his future}

The ongoing blending of the real with the virtual in our lives as seen from an evolutionary perspective is an almost natural response to the technologization of life. Humans rapidly adjust to a changing world and use their knowledge to exploit the environment to the best of their abilities at the time.

It is undoubtedly desirable to reach for more and more in life. The human species builds upon the experiences and achievements of previous generations. Our material culture, using things around us to make tools and solve problems, has made us ultimately who we are - intelligent human beings. Our history started some two million years ago with the earliest tool-maker, Homo habilis [1]. A number of developmental and behavioral changes which have taken place in humanity during its evolution led to the emergence of Homo sapiens, our direct ancestors, some 100,000-150,000 years ago. Finally, we have reached the digital Homo habilis stage of evolution.

Today we are already working on the creation of artificial life and intelligence which mimics human

Manuscript received: Mar. 6, 2014 / revised: Apr. 11, 2014

Corresponding Author: robert@wierzbicki.org

Tel: +49(0)3727-948-146, Fax: +49(0)3727-948-146

University of Applied Sciences Mittweida, Germany 
personality and behavior and which would enable the emergence of high levels of abstract reasoning and inferential thinking in non-human systems of the future. We are undertaking research on the rise of consciousness in machines [12] and combining genetically engineered organic compounds with non-organic-based structures [26]. New techniques like MRI make the study of the human brain and its functions accessible to research [17, 18]. We now have a much better insight into what previously had to compulsorily be approached as a black box. Nothing which has so far been science fiction seems unrealistic anymore. The world becomes more and more comprehensible.

\section{The nature-nurture issue}

There is no doubt that digital technology is influencing how we think and consequently how we behave. Watson claims that the debate is not really whether there is any influence, but rather how certain types of digital technology affect the kinds of thinking [19].Dazzled by a general euphoria about technological convergence and advances, we gradually separate ourselves from nature and from what is (still) perceived as reality. The human values anchored in culture and society become more and more virtualized and overlapped with technical norms, which have become determinants of the "modern" human attributes and paradigms of technological development [24].

Concurrently, in a world of electronic omnipresence and universal changeability, there is a growth in people's longing for socially-responsible integration in the real society, for true and genuine friendship and love, and for the retention of humanitarian values that people have worked hard for centuries to achieve. Time and again, the topic of nature-versus-nurture comes up in discussions [6]. The nature/nurture controversy has been widely discussed in the literature [4-8, 10, and 20]. Richard Dawkins, a renowned English ethologist, evolutionary biologist, author and advocate of atheism, claimed that the topic of nature-nurture as a determinant of human attributes would frequently inspire rather boring writing [7]. But these days the discussion on nature-versus-nurture seems to be more topical than ever. Many people cannot cope with ultra-fast developments in digital society, permanent stress and the fear of failure accompanying our life. They may develop apathy, anxiety, loss of the ability to distinguish between right and wrong and different kinds of challenging behaviors like aggression or self-injury [3, 21], which are typically being additionally reinforced by social events, interactions in advantageous environments and peers of similar attitudes. This development might pose threats for the future human since there is obviously a correlation between complex behaviors and schemes encoded in the human gene networks even if the dynamics of the evolutionary processes and their consequences have not yet been examined in detail:

„In this quest, it is still early days, and it might still be asking too much of neuroscience to provide definitive answers to such contentious issues as the nature-nurture debate in brain development or the relative roles of genetics and environment in human behavior." [10]

\section{Upbringing in the digital age}

The focus when bringing up children is primarily on the achieving of an acceptable status in society and financial security for the future. Accompanying children as they develop their personality in a climate of moral and ethical values is something which is left to the schools. Parents seem to be unable to cope with the active upbringing of their children anymore. The efficient, educational dialogue often fails because of deficits in the psychological contact with the children. Parenting measures are often based on experiences of parenting methods from one's own childhood. However, these are no longer suitable in today's world. We talk about a conflict of generations [22].

Convergence has changed the way we think about education and especially nurture. But we mistakenly believe that digitally supported education and computer-mediated communication will solve the problems which have accumulated within societies over time. Social networks and social learning are not the panacea. "We must fight against the convenience of the digital era" [19].Social communities help aggregate knowledge and support learning from each other but there is no guarantee that something like an objective conscience or objective, correct rules emerge out of the collectiveness. The behavior of the individual can be adapted to the collective behavior and vice versa. It may however not correspond to any objective values or 
normative foundations for doing the right things, as seen by society. The decision which enjoys the group's consensus may not automatically be the best. The desire for consensus can dominate the individual sense of reason and lead to irrational decisions (i.e. the desire for harmony in a decision-making group overrides a realistic appraisal of alternatives) [13]. Collective cyber bullying might be here a good example.

\section{Games as means of nurturing}

Education refers to the transfer of knowledge by means of teaching and as such it primarily refers to cognition-related aspects in pedagogy. Nurture can be understood as the act of bringing up, in which life-competences are paramount. The use of computer technology in education has been proven to improve learning results and experience especially in the so-called blended environments. "Blended" environments consist of a number of components. The most important are moderated face-to-face methods and computer-mediated activities.

In the last few years the phenomenon of gamification has experienced a major boom. An ever-increasing number of tasks and processes in various parts of our lives - in private, at school, or at work - are formed by mechanisms from the world of games [14]. In this way a new quality of emotion, acceptance, perception and mood develops [15]. Shaped by social media and technological development, games are now part of our contemporary digital culture. Including playing games in curricula seems to be a very promising approach.

As discussed in [22] with appropriate game systems a range of stories can be told and sensitive scenarios can be acted out. By role playing, switching from the actor's position to the observer's position the role behavior of individuals and groups can be called into question and the effects of their actions can be reflected on emotionally. Metaphor-based game design can be an effective tool in understanding and correcting human behavior, especially in pre-adolescence. Metaphor, in particular behavioral metaphor, is a topic dealt with relatively marginally in education studies [23]. Reasoning by analogy is generally considered to be a core component of human cognition [11]. Even children of pre-school age can already form complex analogies [Brown]. Young children's mental models determine analogical transfer across problems with a common goal structure. In the translation of various real-life situations to game-based scenarios, primarily two challenges must be developed for the player: (1) make a decision (e.g. resist a temptation) and (2) bear the consequences of this decision, which cannot be foreseen in every case. Looking at the causes of an action, evaluating its consequences and mapping the outcomes to other situations seems to be a powerful means of nurturing and fostering personal responsibility and respect for others [23].

Positive educational effects of providing content with the help of games cannot, however, be brought about on their own. They can be effectively initiated with the help of a game, but they need the intervention of a moderator (teacher, coach) with whom the events of the game can be analysed and discussed within the group of players. The coach can, if necessary, qualify and correct the perception of game situations.

\section{Summary}

In this paper I have emphasized the role of nurture games with a primary focus on behavior modeling and as part of a virtual nurturing environment, within the frame of convergence of life with technology. It is urgently necessary to adopt a position in the process of upbringing using correct patterns - values and norms - and to adopt new methods of communicating them in pedagogy. This is important so as to build real social and life skills in children and adolescents and to better prepare them to predict the effects of their behavior and decisions. The overall goal is to alleviate the effects of ego threats at the conscious and unconscious level. Decisions in life may be irreversible, and their consequences can be very serious, leading to psychological tension and trauma. Young people often perform actions led by temporary fun and spontaneous, emotional attitudes, or which emerge out of peer-pressure. These actions often lack any rationality and they may have tragic consequences the youngsters are not aware of.

At the University of Applied Sciences in Mittweida, Germany, a series of psychological tests utilizing behavioral metaphors are currently (2013) under development. The tests provide the basis for more complicated scenarios in multiple player games. The problem is that the design of nurture games requires cooperation between the media sector, psychologists and game designers, and it is not always easy to bring 
all of these together. This is where the convergence of life and technology is extremely valuable and desired.

Game publishers are sceptical when it comes to bringing a game onto the market which has more psychological depth [20]. In today's world everything is geared towards generating income and games with an educational focus just don't sell well, if at all. For this reason more commitment is needed by educational institutions and ministries. It is only then that we will be in a position to preserve human values in society.

\section{Acknowledgement}

This paper was completed within the framework of the project PrävEm [25] supported by The European Social Fund (ESF) and the Saxon Ministry of Science and the Fine Arts (SMWK) in 2013.

\section{References}

[1] Agutter P. S., Wheatley D. N., "About Life. Concepts in Modern Biology", Springer, 2007.

[2] Brown A. L., Kane M. J., \& Echols C. H., "Young children's mental models determine analogical transfer across problems with a common goal structure", Cognitive Development, 1, 103-121, 1986.

[3] Cooper P., Smith J. S., Upton G., Emotional and behavioural difficulties: theory to practice", Routledge, London, 1994.

[4] DawkinsR.,"Good strategy or evolutionarily stable strategy?" In "Sociobiology: Beyond Nature/Nurture" (eds. G. W. Barlow and J. Silverberg), Boulder, Colorado: Westview Press. pp. 331-67, 1980.

[5] DawkinsR.,"The Ancestor's Tale: A Pilgrimage to the Dawn of Evolution", Weidenfeld \& Nicolson, London, 2004.

[6] DawkinsR.,"The Selfish Gene: 30th Anniversary edition", Oxford University Press, 2006.

[7] Dawkins R., "Modern Science Writing", Oxford University Press, 2008.

[8] Dobzhansky T, "Mankind Evolving", Yale University Press, 1962.

[9] DoidgeN.,"The Brain That Changes Itself: Stories of Personal Triumph from the Frontiers of Brain Science", Penguin Books, New York, 2007.

[10] Dowling J. E.,"The great brain debate: nature or nurture?", Joseph Henry Press, 2004.

[11] Goswami U.,"Analogical Reasoning in Children", Hillsdale, NJ: Lawrence Erlbaum Associates, 1992.

[12] Haikonen P.O., "Robot Brains: Circuits and Systems for Conscious Machines", John Wiley \& Sons, Ltd. 2007.

[13] Janis I. L., "Victims of Groupthink: A psychological study of foreign policy decisions and fiascoes", Houghton Mifflin, Boston, 1972.

[14] Kapp, K. M., "The Gamification of Learning and Instruction: Game-based Methods and Strategies for Training and Education", John Wiley \& Sons, 2012.

[15] Keitt T. J.,"It’s Time to Take Games Seriously", MA: Forrester Research, Cambridge, 2008.

[16] Miller H. C. "Digital Storytelling: A Creator's Guide to Interactive Entertainment", Butterworth Heinemann, 2008.

[17] Soon C. S., Brass M, Heinze H. J., Haynes J. D., "Unconscious determinants of free decisions in the human brain", Nat Neurosci., May;11(5):543-5, 2008.

[18] Stroman P. W., "Essentials of Functional MRI", CRC Press, Taylor \& Francis Group, 2011.

[19] WatsonR., "Future Minds: How the Digital Age is Changing Our Minds, Why this Matters and What We Can Do About It", Nicholas Brealey Publishing, 2010.

[20] Wilson E.O., "A consideration of the genetic foundation of human behavior", in G. W. Barlow and J. Silverberg (eds.), "Sociobiology: Beyond Nature/Nurture? AAAS Selected Symposium 35", Boulder, CO: Westview Press, 295-306, 1980.

[21] Wierzbicki R.J., Kermer K., Röbisch K, Schmieder T., Straßburger T., "Media Pedagogics in Converged Environments of the Future", Proc. iTV 2010, Tampere, Finland, 2009.

[22] Wierzbicki R. J.,"Blended Nurture", in T.-h. Kim et al. (Eds.): ASEA/DRBC/EL 2011, CCIS 257, pp. 643-650, 2011. Springer-Verlag Berlin Heidelberg, 2011.

[23] Wierzbicki R. J., Boehnke P., "Behavioural Metaphors for Nurturing through Computer Games", to be published.

[24] Vermaas P., Kroes P., van de Poelm I., Franssen M., and Houkes W., "A Philosophy of Technology - From Technical Artefacts to Sociotechnical Systems", Morgan \& Claypool, 2011.

[25] Project PrävEM: "Enterprise Content Management-based Production Pipelines for 3D-Pre-Visualisation and Animated Film", http://www.hs-mittweida.de/praevem/(October 29, 2011).

[26] http://www.dailymail.co.uk/sciencetech/article-2050500/Biological-computers-soon-reality-scientists-build-basiccomponents-bacteria-DNA.html(October29, 2011). 